\title{
Design of fault diagnosis expert system for electronic control system of adaptive optics based on Fault Tree
}

\author{
Guoqing Gao ${ }^{1,2,3, a}$, Luchun Zhou ${ }^{1,2, b^{*}}$ \\ ${ }^{1}$ Key Laboratory on Adaptive Optics, Chinese Academy of Sciences, Chengdu, Sichuan, China \\ ${ }^{2}$ Institute of Optics And Electronics, Chinese Academy of Sciences, Chengdu, Sichuan, China \\ ${ }^{3}$ University of Chinese Academy of Sciences, Beijing, China \\ a296405364@qq.com, b907570582@qq.com,
}

\section{Keywords: AO Expert System Fault Diagnosis FTA}

Abstract. In view of the complexity of the electronic control system of adaptive optics, combining with fault tree analysis and expert system, we design an expert system on fault diagnosis for electronic control system of adaptive optics based on Fault Tree. After analysis of electric control system of AO, fault tree can be established. The expert system is the extensive application of the fault tree. The system uses forward inference method to diagnose failure of system. In addition to traditional expert systems, point of failure was set to obtain real-time fault information. Then the information was sent to diagnosis expert systems to get the online function of diagnosis of system. The system highly improved the stability of AO system.

\section{Introduction}

Adaptive Optics (AO) technology is actually the automatic control system targeting on optical wavefront. It goes through real-time measuring-controlling-correcting dynamic error of optical wavefront (is shown in Figure 1) make the optical system have the ability to overcome dynamic error. The electric control system of the AO covers the acquisition, processing, correction, imaging, communications and other parts of the AO system. So, the stability of electric control system is a key point of the AO system.

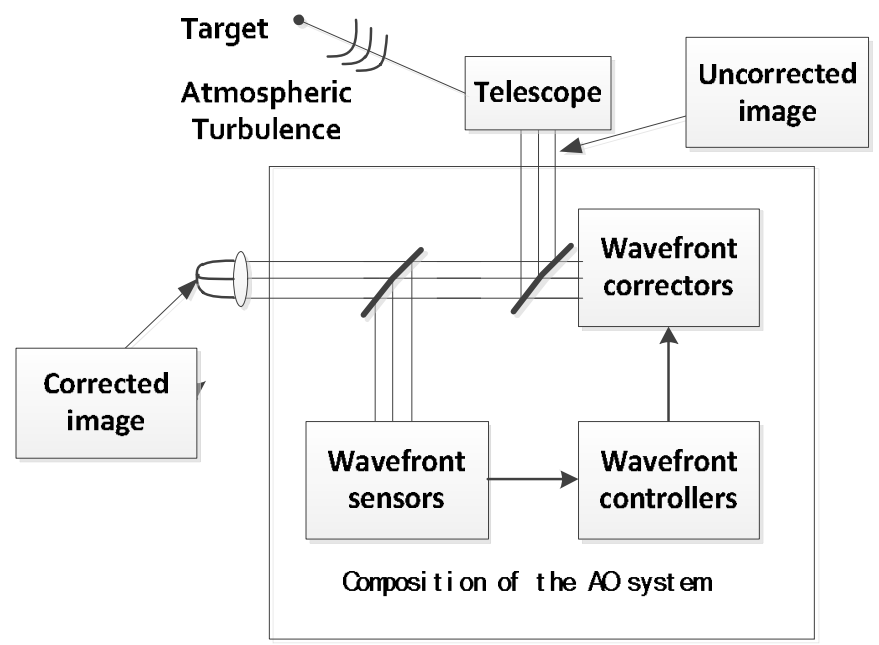

Figure 1 System schematic of adaptive optics

Adaptive optics technology is a new optical technology developed in recent 40 years. In China, the study of adaptive optics has started earlier. So applications of adaptive optics system are increasingly widespread. As the developing of adaptive optics system, people come to realize that the stable adaptive optics system is also an unavoidable topic when we upgrade the system. However, the current study of fault diagnosis system of adaptive system and electronic control system of adaptive optics system is very rare. 
This paper combines the fault tree analysis (FTA) and fault diagnosis expert system to design fault diagnosis expert system of electric control system of AO .It provides a guarantee for the stable work of the electronic control system of $\mathrm{AO}$ and $\mathrm{AO}$ systems.

\section{Structure of expert system}

The fault diagnosis expert system of electronic control system of AO based on fault tree is shown in Figure 2. At first, analysis of AO system and electronic control system of AO is necessary. Then we use the FTA to obtain knowledge of fault. By this way, expert system knowledge base will be added and improved. What's more, point of failure will be set to obtain fault information of electronic control system of AO. In addition to the conventional expert system, point of failure makes the system have the function of self-checking and real-time online diagnosing. Users send diagnosis instructions in the human-computer interaction interface via Ethernet. Then, the point of failure will return the fault information to the expert system after receiving the instruction. This information will help the expert system to judge the fault and help users to solve the problem.

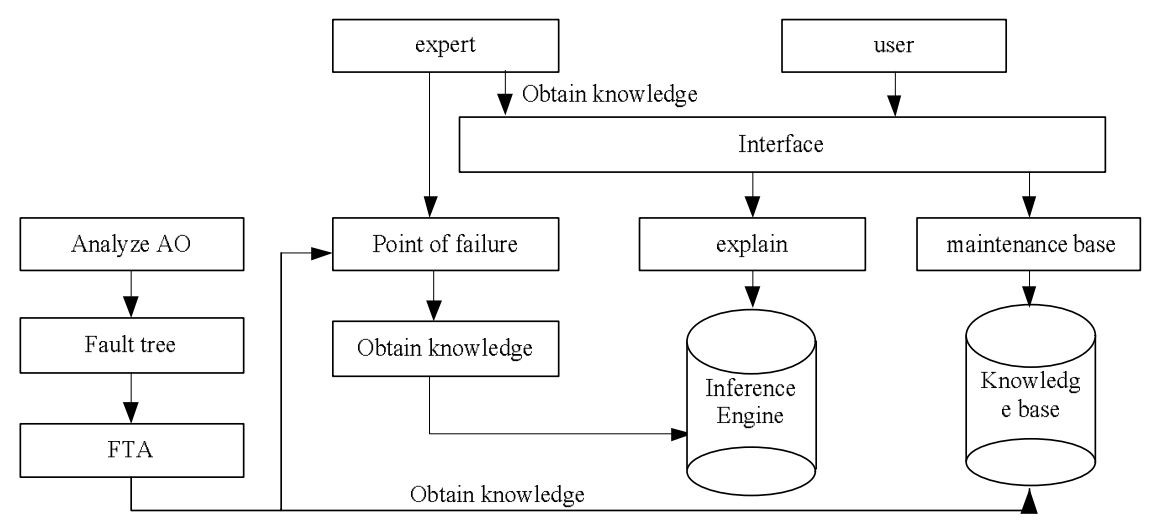

Figure 2. The component of expert system

In addition to setting the point of failure, the traditional expert system also includes explanation mechanism, knowledge acquisition and management, knowledge base, inference engine, human-computer interaction module, etc. Knowledge base is for storing specific fault knowledge. Knowledge acquisition and management module is mainly responsible for storing the fault information in the knowledge base in certain patterns. Knowledge acquisition and management also can add, delete, and modify the data of knowledge base. Inference engine module is used for simulating the thinking of expert. It can solve the fault according to the fault information and knowledge from knowledge base under certain rules. Explanation mechanism will explain the process of the work of Inference engine and guide the user to troubleshoot problems.

\section{Knowledge Base}

Fault Knowledge Acquisition. When design fault diagnosis expert system of electric control system of AO, the first problem should be solved is to establish fault diagnosis model and obtain fault knowledge as far as possible. Analysis of failure by FTA can simply and conveniently analyze system failure. It is very helpful to solve the problem of obtaining knowledge of failure.

Fault tree analysis is a kind of top-down graphics deductive analysis method. Methods to establish the fault tree are deductive and synthesis. Deductive method is mainly used for establishing the fault tree by human. Synthesis is mainly used for establishing the fault tree by computer. Using deductive method to generate a fault tree requires the designer be fully aware of the various components of system. After analyzing the electronic control system of $\mathrm{AO}$ and summering engineering experience, the fault tree of electric control system of the AO can be divided into failure of wavefront system, failure of power 
system, failure of communication system, failure of the imaging system. It is shown in figure 3 . The description of fault is shown in Table 1.

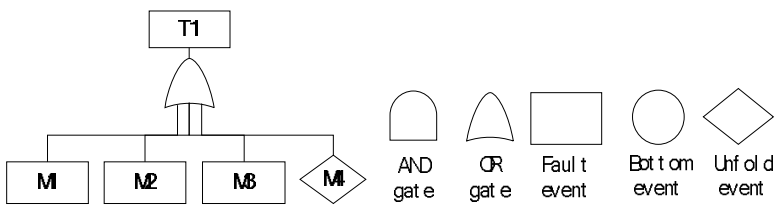

Figure 3 Fault tree of electronic control system of AO

Table 1 Description of fault No.for Figure 3

\begin{tabular}{|c|c|c|c|}
\hline Fault No. & Fault Name & Fault No. & Fault Name \\
\hline T1 & Fault of electrical System of AO & M1 & Fault of system monitoring server \\
\hline M2 & Fault of imaging monitoring server & M3 & Fault of communication system \\
\hline M4 & Fault of power system & & \\
\hline
\end{tabular}

Due to the complexity of the electronic control system of AO and fault models, we choose fault tree of wavefront system to analyze the fault of system.It is shown in Figure 4. The description of fault is shown in Table 2.

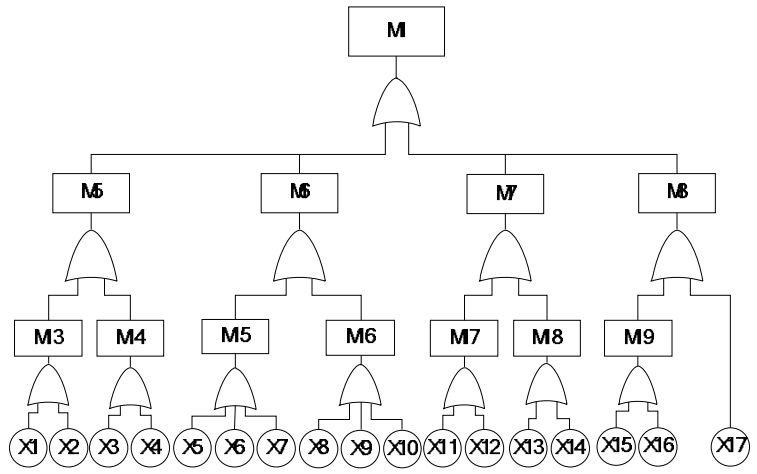

Figure 4 Fault tree of wave front system of AO

Table 2 Description of fault No. for Figure 4

\begin{tabular}{|c|c|c|c|}
\hline Fault No. & Fault Name & Fault No. & Fault Name \\
\hline M5 & Fault of wavefront detection system & M6 & Fault of wavefront processing system \\
\hline M7 & Fault of wavefront controlling system & M8 & Fault of wavefront correcting system \\
\hline M13 & Fault of precision tracking(PT) sensor & M14 & Fault of Shack-Hartmann Sensor(S-H)sensor \\
\hline M15 & Fault of PT processor & M16 & Fault of S-H processor \\
\hline M17 & Fault of PT HVA & M18 & Fault of S-H HVA \\
\hline M19 & Fault of S-H correction system & X1 & Fault of S-H sensor receiving \\
\hline X2 & Fault of PT sensor transmitting & X3 & X5 \\
\hline X4 & Fault of S-H sensor transmitting & PT processor parameter is not loaded correctly \\
\hline X6 & Fault of PT processor & X7 & Fault of PT processor transmitting \\
\hline X8 & S-H processor parameter is not loaded correctly & X9 & Fault of S-H processor \\
\hline X10 & Fault of S-H processor transmitting & X11 & Fault of PT HVA receiving \\
\hline X12 & Fault of PT HVA power returning & X13 & Fault of S-H HVA receiving \\
\hline X14 & Fault of S-H HVA power returning & X15 & Fault of S-H TM receiving \\
\hline X16 & Fault of DM receiving & X17 & Fault of PT TM receiving \\
\hline
\end{tabular}

The main purpose of fault tree analysis is to find the minimal cut sets of fault tree, calculate the degree of importance of the minimal cut sets and failure probability of the top event. We use Fussell method to find minimal cut sets and go as follows. $\mathrm{K} 1:\{\mathrm{X} 1\}, \mathrm{K} 2:\{\mathrm{X} 2\}, \mathrm{K} 3:\{\mathrm{X} 3\}, \mathrm{K} 4:\{\mathrm{X} 4\}, \mathrm{K} 5:\{\mathrm{X} 5\}, \mathrm{K} 6$ : $\{\mathrm{X6}\}, \mathrm{K} 7:\{\mathrm{X} 7\}, \mathrm{K} 8:\{\mathrm{X} 8\}, \mathrm{K} 9\{\mathrm{X} 9\}, \mathrm{K} 10\{\mathrm{X} 10\}, \mathrm{K} 11:\{\mathrm{X} 11\}, \mathrm{K} 12:\{\mathrm{X} 12\}, \mathrm{K} 13:\{\mathrm{X} 13\}, \mathrm{K} 14:$ 
$\{\mathrm{X} 14\}, \mathrm{K} 15:\{\mathrm{X} 15\}, \mathrm{K} 16:\{\mathrm{X} 16\}, \mathrm{K} 17\{\mathrm{X} 17\}$. Electronic control system of AO has been put into use. Because of the high stability of the system, we assume that failure probability of each bottom incident according to engineering experience is

$p_{x}(5)=p_{x}(8)=p_{x}(11)=p_{x}(12)=p_{x}(13)=p_{x}(14)=1.5 * 10^{-5}$, the others are $p_{x}(i)=10^{-5}$, Undeveloped events are $P_{m}(i)=10^{-4}$. So according to the formula

$$
I_{q}(i)=\frac{\partial g}{\partial p_{\mathrm{N}}(\mathrm{i})},
$$

$g$ is probability of top event.

We get the importance of the minimal cut sets as follows:

$I_{q}(5)=I_{q}(8)=I_{q}(11)=I_{q}(12)=I_{q}(13)=I_{q}(14)>I_{q}(1)=I_{q}(2)=I_{q}(3)=I_{q}(4)=$

$I_{q}(6)=I_{q}(7)=I_{q}(9)=I_{q}(10)=I_{q}(14)=I_{q}(15)=I_{q}(16)=I_{q}(17)$

Knowledge Representation. The expert system is based on IF ... THEN ... rules, such as:

IF Fault of PT TM receiving;

THEN Fault of wavefront correcting system.

In the fault tree model, if we regard the top event as the fault which need expert system to analyze and solve, the bottom events will be the result of expert system. So the hierarchy of the fault tree corresponding to process expert system inferring. The cut set contact Fault Tree and Expert System knowledge base. So, a cut set of fault tree is a kind of failure mode of electronic control system of AO. In rule-based expert system, cut sets correspond to rules of knowledge base. And the conclusions of rules are the bottom events of cuts.

Knowledge base is the key module of the expert system. The level of knowledge will impact on the efficiency of the inference engine and maintenance of knowledge base directly. Fault tree of electronic control system of AO has been discussed. Converting these fault trees to knowledge of the rules of knowledge base is not enough to constitute the entire contents of the expert system. So we need to add additional information such as troubleshooting methods of fault, location of fault, features values of fault, etc. Therefore, in order to manage and maintain the knowledge base, knowledge base will express as rules table, features table and conclusions table. Rule table use for storing rule information of fault which extracted from fault tree. Features table use for storing features value of fault. Conclusion table mainly use for storing solutions of fault.

\section{Inference Engine}

Inference engine is the core of the expert system. Diagnosis flow of expert system is shown in the figure 5. The paper mainly uses the forward inference. The system mainly accord characteristic values extracting from what users input and the status of point of failure to match the feature value from knowledge base. If success, the system will diagnose the fault as well. If fail, the designer will update knowledge base and set the point of failure to make the system have the function of learning. Fault information from the point of failure can help system to narrow range and simplify diagnostic procedures according to information of failure location. For the information from user, we can follow step by step to match fault tree to complete diagnosis finally. 


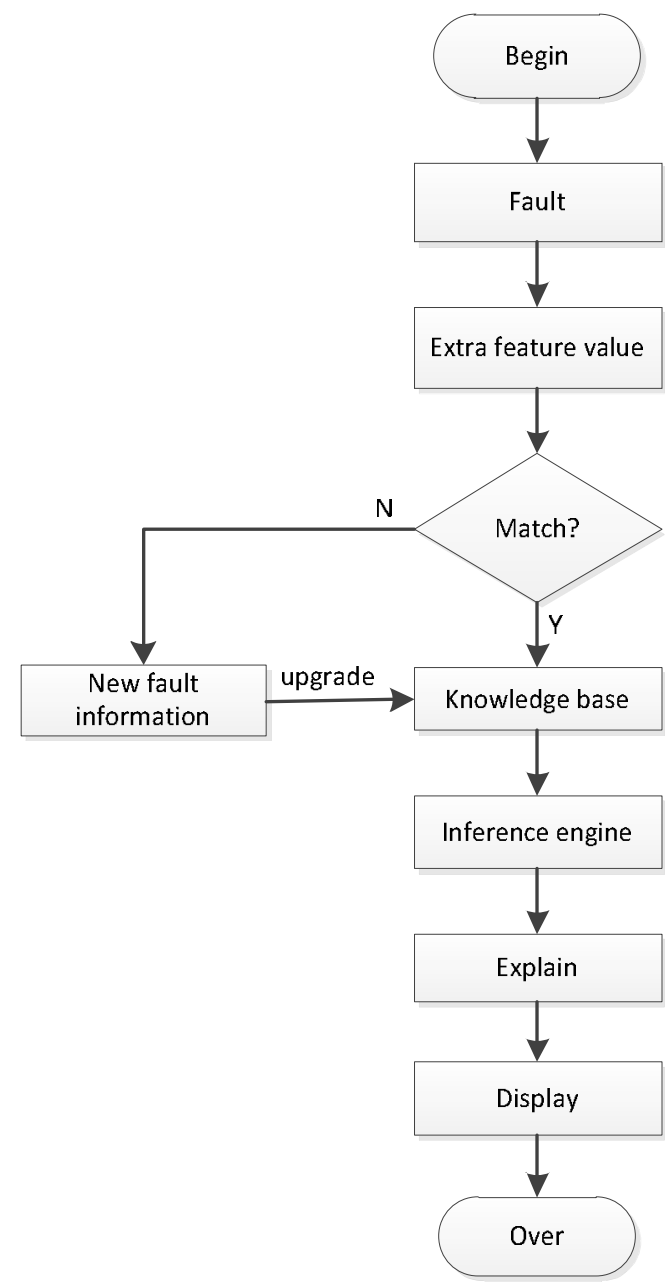

Figure 5 The Flow of Inference Engine

\section{Summary}

The paper presents a design method of fault diagnosis of electric control system of AO. The article combine characteristics of electronic control system of $\mathrm{AO}$ and engineering experience to analyze the system by the FTA. It is all-round and intuitive to describe system failures. It is a good solution to the problem that it is hard to obtain knowledge of expert system. System introduces on-line diagnosis on the basis of expert system to test system real-time. The system is good to improve the intelligence level of electronic control system of AO. And it is useful for stability of AO system.

\section{References}

[1] Zhang Qi , Liao Jie , Wu Jianjun, LIU Yu2, Design of Diagnostic Expert System for Electronic System of General Equipment Based on FTA, ACTA ARMA MENTARII. 2008, 29-2.

[2] Zong Qun, Li Guangyu, Guo Meng, Design of Diagnostic Expert System for Elevator System Based on FTA, Control Engineering of China, 2013,20-2.

[3] Zhang Xi, Du Xusheng, Liu Chaoying, Development of Railway Station Signaling Control Equipment Fault Diagnosis Expert System, Journal Of The China Railway Society, 2009, 31-3

[4] Jiang Wenhan. Adaptive Optics Technology, Chinese Journal of Nature, Invited Special Paper 28-1 